\title{
Association of Amino Acids Embedded in Helium Droplets Detected by Mass Spectrometry.
}

\author{
Matthieu R. Lalanne ${ }^{\text {a }}$, Georg Achazi, Sebastian Reichwald, and Albrecht Lindinger \\ Institut für Experimentalphysik, Freie Universität Berlin, Arnimallee 14, 14195 Berlin, Germany
}

\begin{abstract}
Amino acids were embedded in helium droplets. The electron impact ionization allows for detecting positively charged glycine, valine, histidine, tryptophan and their principal fragments. Monomers and polymers with up to four amino acids are reported. Heterodimers of tryptophan and valine or histidine are observed as well as heterodimers of included fragments. The ability of these associations of molecules to form complexes with water is examined.
\end{abstract}

Amino acids are considered as elementary bricks of life. Peptide bonding between proteogenic amino acids constitutes a vector of genetic expression in cells. The detection of amino acids on meteorites [1] and comets [2] warrants examination as their possible association in gas phase is of vast scientific interest. Helium nanodroplets have the advantage of generating an isolation matrix in which the molecules conserve quasicompletely the vibrational and rotational freedom of the gas phase [3]. At temperatures below 2.17 Kelvin, liquid helium $\left({ }^{4} \mathrm{He}\right)$ adopts the superfluidity state, which corresponds to a fluid with zero viscosity. Through evaporative cooling via expansion in vacuum, helium nanodroplets reach a temperature of $0.38 \mathrm{~K}$, which corresponds to their superfluidity state [4]. Nanodroplet beams allow for embedding and isolating all molecules coming into contact with the helium. In the droplets clusters of embedded molecules are suspended [5]. In this article the attempt to embed several different amino acids in helium droplets is reported. Monomers as well as oligomers of amino acids are observed. The simultaneous pickup of different amino acids also allows the observation of heterodimers. The amino acids involved in this experience include glycine (gly), valine (val), histidine (his) and tryptophan (trp). Glycine is the smallest amino acid, in which the lateral chain is composed only of one hydrogen atom. Tryptophan is the heaviest proteogenic amino acid, composed of an indole cycle in the lateral chain. Histidine includes an imidazole cycle and valine a methylbutan on the lateral chain. Several previous studies already reported glycine $[6,7]$, valine [8], histidine and tryptophan [9] embedded in helium droplets. In this article, the ability of amino acids to form heteropolymers with other amino acids, fragments of amino acids and water is examined and enhance the information about the complex formation in helium droplets.

\section{Experimental setup}

The experiment was performed with a supersonic beam of ${ }^{4} \mathrm{He}$ achieved through a $5 \mu \mathrm{m}$ nozzle cooled to $12 \mathrm{~K}$ at a pressure of 25 bars. This supersonic beam passes through a pick up cell that is designed with apertures of 2 and 3 millimeter diameter (respectively upstream and downstream). The pickup cell contains few milligrams of the amino acids, which are vaporized by a heating element allowing temperatures up to $200^{\circ} \mathrm{C}$. The $10-\mathrm{mm}$ path through the cell remains long enough to permit the pickup of the amino acids of interest. The nanodroplet constituents are detected in their positively charged form by a Balzers QMG-420 quadrupole mass analyzer (mass resolution $\mathrm{m} / \Delta \mathrm{m} \approx 1000$ ) after the electron impact ionization performed by the source set at a voltage of $50 \mathrm{~V}$ (figure 1).

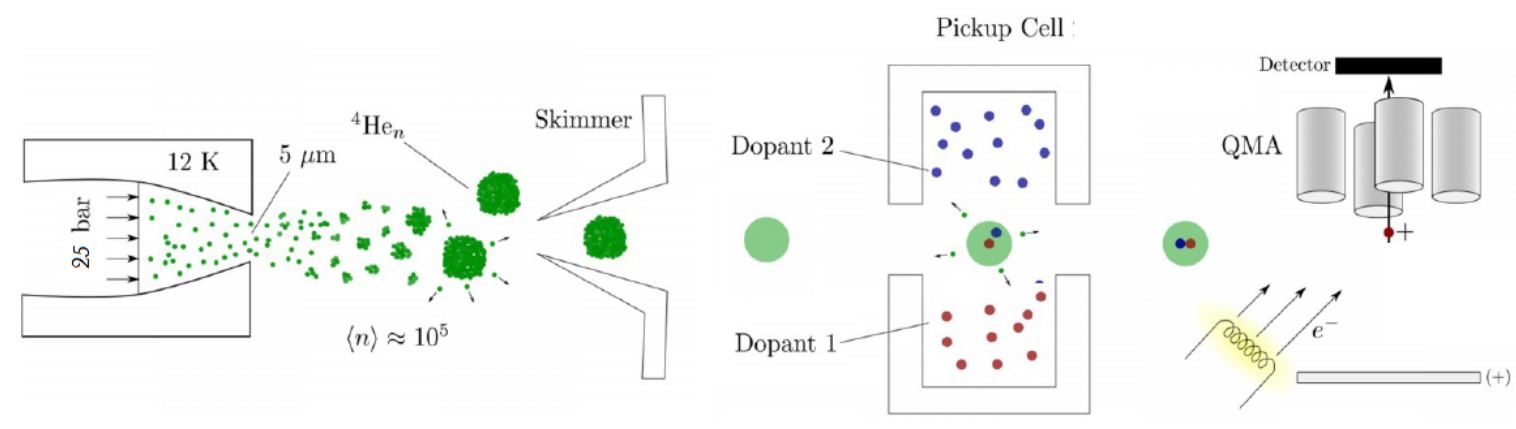

Figure 1: Illustration of the helium droplet experimental setup. Amino acids represent the dopant investigated here. Picture adapted from [9]. 


\section{Results and discussion}

\subsection{L-glycine}

Embedded glycine is detected in its protonated form [gly, H] at $76 \mathrm{~m} / \mathrm{z}$. The amino acids are introduced in the pickup cell in a crystalline form. In this form the amino acids include water molecules, which vaporize by the heating of the pickup cell and allow for observation of glycine, also in complexes with water molecules. Figure 2 shows glycine complexes composed of up to 14 water molecules (where glycine remains in its protonated form). Complexes comprised of four water molecules and eight water molecules are clearly favored. The signals at $148 \mathrm{~m} / z$ [Gly, $\mathrm{H}, 4 \mathrm{H}_{2} \mathrm{O}$ ] and at 220 $\mathrm{m} / z$ [Gly, $\mathrm{H}, 8 \mathrm{H}_{2} \mathrm{O}$ ] are detected in greater quantity than their homologues with either one more or one less water molecule. Complexes formed with only one water molecule and a hydrogen nucleus exhibit a series of clusters. These water clusters clearly favored five- and ten-water-molecule groupings. In figure 2 the detections at $91 \mathrm{~m} / z\left[5 \mathrm{H}_{2} \mathrm{O}, \mathrm{H}\right]$ and at $181 \mathrm{~m} / z\left[10 \mathrm{H}_{2} \mathrm{O}, \mathrm{H}\right]$ are more abundant than the other clusters in the series, which correspond to complexes with either one more or one less water molecule. In the gas phase the structure of $\mathrm{H}^{+} /\left(\mathrm{H}_{2} \mathrm{O}\right)_{n}$ revealed a first shell of water molecules by $n=4$ and subsequent shells which form clathrate cages with $n=21$ and $n=28$ [10,11]. The configuration of water clusters in helium droplets appears to remain different in comparison to the configuration in the gas phase.

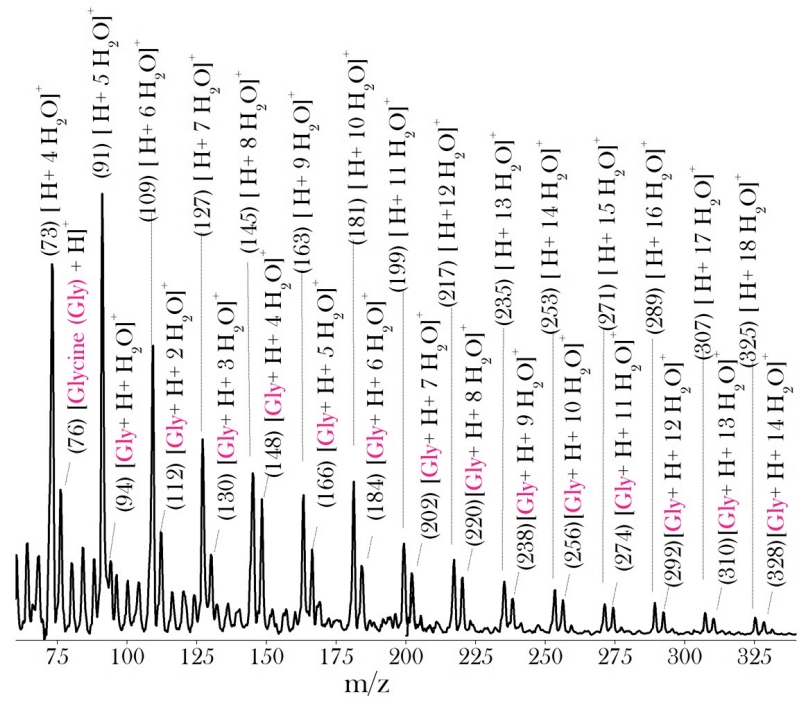

Figure 2: Mass spectrum of embedded molecules, which results from a helium droplet beam passing through the pickup cell containing glycine.

By itself, a unit composition of five water molecules is favorably detected. However, when added to protonated glycine, a unit composition of four water molecules is observed in great quantity. Amino acids experienced fragmentation through the electron impact ionization, particularly in their immonium ions. These ions correspond to the amino acids truncated from the carboxyl functional group $[-\mathrm{COOH}]$ (which represents fewer than $45 \mathrm{amu}$ ). The immonium ion of glycine is observed at $30 \mathrm{~m} / \mathrm{z}$, a low concentration in comparison to glycine (figure 3 ).

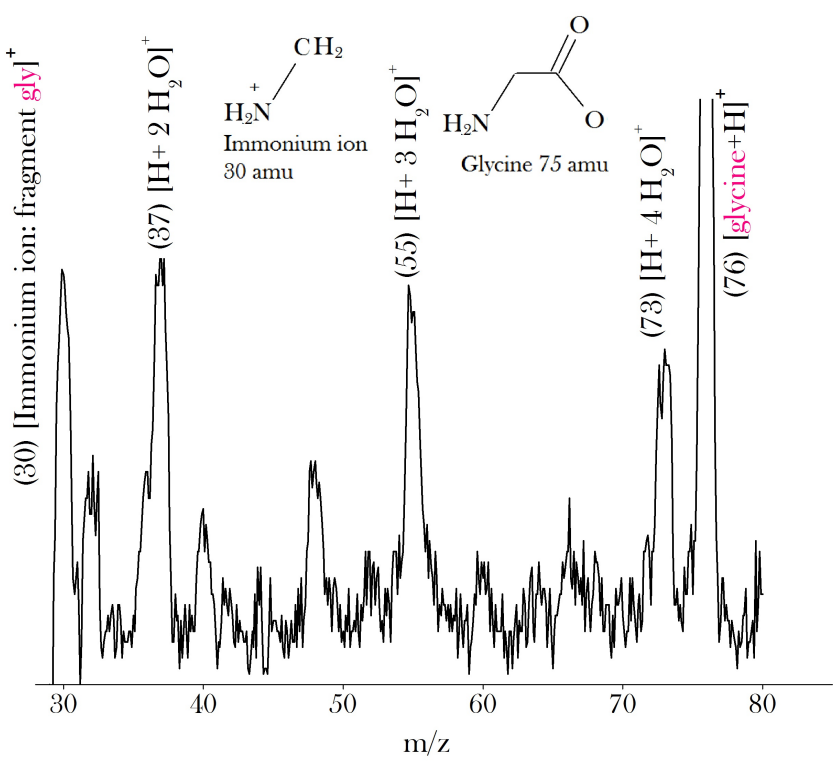

Figure 3: Mass spectrum of embedded molecules, which results from a helium droplet beam passing through the pickup cell containing glycine in the presence of fewer water compared to the spectrum presented in figure 2. A fragment of glycine is also detected.

Electron impact ionization on helium droplets causes fragmentation of the embedded molecules through different processes than in the gas phase $[12,13]$. Ionization of a helium droplet forms a migrating $\mathrm{He}^{+}$hole, which releases substantial energy and provokes fragmentation in the molecules. This fragmentation could be dramatically reduced by the presence of water in helium droplets. Different processes, which explain the protection caused by the water molecules, are suspected. A buffering effect, which originates from a solvation surrounding the fragile molecule, is presumed. A study, which compares the fragmentation of glycine, polyglycine, alkanes and alkanethiols, proposes a concurrent process [6]. This study shows protection from molecular fragmentation by water molecules, which is particularly present when the embedded molecules exhibit a low electric dipole moment. The solvation remains favored by molecules that possess a large dipole moment. The suggestion is made that migrating $\mathrm{He}^{+}$holes are steered by water complexes located near fragile molecules. This protection originating from the water molecules remains limited when the fragile molecules possess a high dipole moment 


\subsection{L-valine}

Several embedded polymers of valine are observable in helium droplets (figure 4). Valine monomers are detected in the protonated form at $118 \mathrm{~m} / \mathrm{z}$. Monomers are observed in complexes, which contain up to 9 water molecules (figure 3). Polymers with up to four valine molecules are detected with the added presence of a hydrogen nucleus. The fragment of valine, which corresponds to its immonium ion, is also detected at $72 \mathrm{~m} / \mathrm{z}$. The peak of this fragment presents an asymmetry, which can be explained by the presence of $[4$ $\left.\mathrm{H}_{2} \mathrm{O}, \mathrm{H}\right]$ at $73 \mathrm{~m} / \mathrm{z}$. The complexes composed of valine and four water molecules could be interesting regarding the particular distribution of water complexes observed for glycine with water. Unfortunately, the [valine, $\mathrm{H}, 4 \mathrm{H}_{2} \mathrm{O}$ ] share the same mass than complexes made of valine, its fragment and a hydrogen nucleus observable at $190 \mathrm{~m} / \mathrm{z}$. This is also the case for the polymers of valine and four molecules of water, which are not distinguishable from complexes including a fragment of valine. However, a previous study on valine/water sub-clusters in helium droplets showed a monotonic decrease and no intensity anomalies at all [8]. Dimers made of the valine fragments $(72 \mathrm{amu})$ are not observed and thus the importance of carboxyl functional groups (C-terminus) in the formation of the complexes of valine could be suspected.

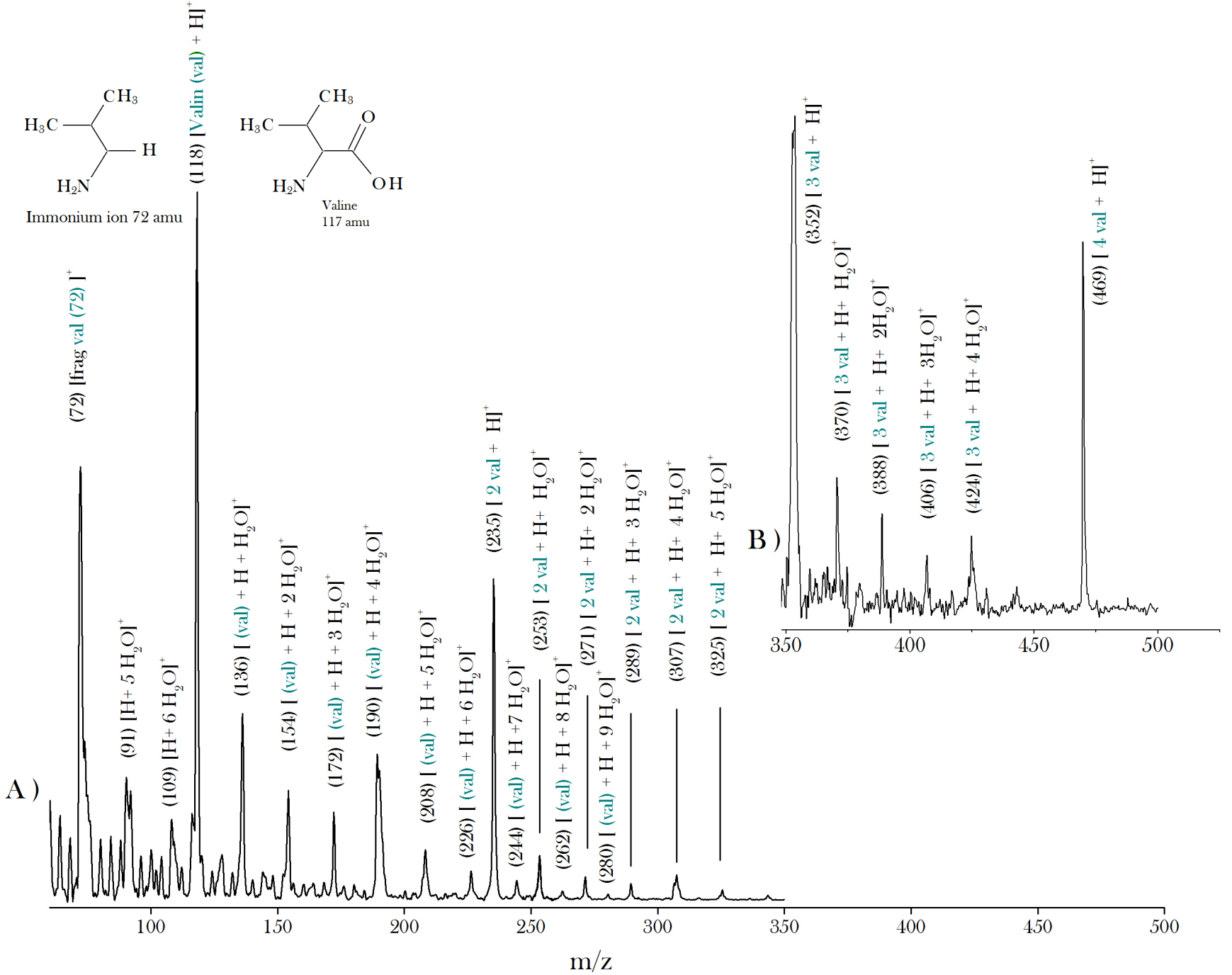

Figure 4: Mass spectra of embedded molecules, which result from a helium droplet beam passing through the pickup cell containing valine. The mass spectra are obtained through the difference of mass spectra in presence and absence of helium droplets. The two spectra A) and B) were recorded with different signal amplification. 


\subsection{L-valine and L-tryptophan}

In order to embed simultaneously two different amino acids, valine and tryptophan are introduced in the pickup cell. The vaporization temperature of valine is lower than that of tryptophan. However the simultaneous detection of both amino acids remains possible (figure 5). Valine monomers in their protonated form at $118 \mathrm{~m} / z$ are also detected in complexes with water molecules. In these conditions, the favored complexes at $190 \mathrm{~m} / \mathrm{z}$ (observable in figure 4) are not remarkable anymore. Tryptophan monomers are detected at $204 \mathrm{~m} / z$ and also form complexes with water molecules. The characteristic fragments of tryptophan are observed at 130 $\mathrm{m} / \mathrm{z}$ and $159 \mathrm{~m} / \mathrm{z}$. The small fragment corresponds to the tryptophan, where the amide functional group [CH- $\mathrm{NH}_{2}-$ $\mathrm{COOH}]$ remains truncated. The larger fragment corresponds to tryptophan without the carboxyl functional group [COOH]. Neither fragment is detected in complexes with water molecules. A bond with water molecules located on the carboxyl functional group (C-terminus) of the amino acids could be suggested.

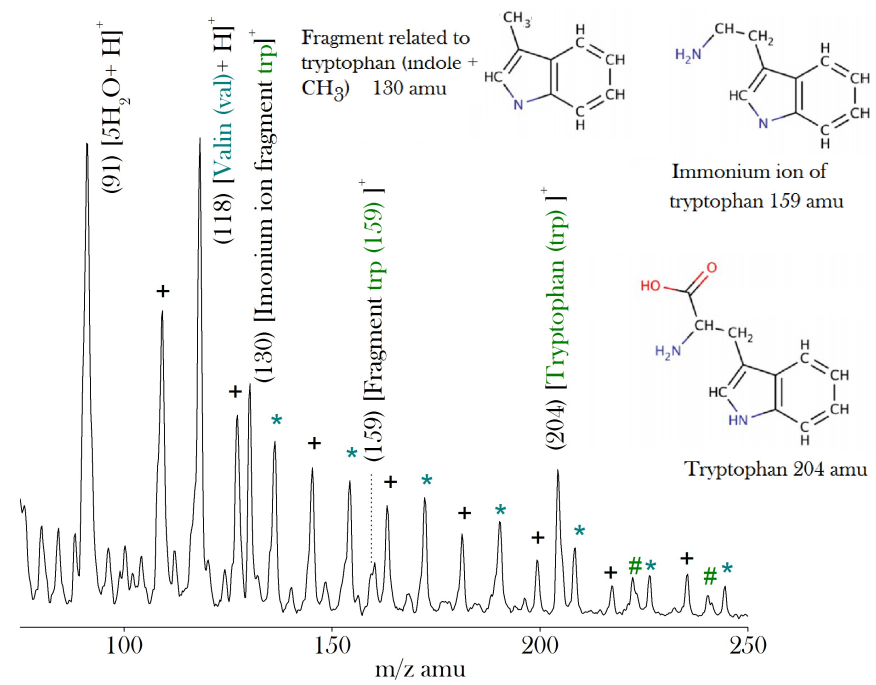

Figure 5: Mass spectrum of embedded molecules, which results from a helium droplet beam passing through the pickup cell containing both valine and tryptophan. The mass spectrum is obtained through the difference of mass spectra in presence and absence of helium droplets. The sign + corresponds to the $\left[\mathrm{H}, \mathrm{n} \mathrm{H}_{2} \mathrm{O}\right.$ ], whereas * and \# correspond to [val, $\mathrm{H}, \mathrm{n}$ $\mathrm{H}_{2} \mathrm{O}$ ] and [trp, $\mathrm{n} \mathrm{H}_{2} \mathrm{O}$ ], respectively. The molecular structures were adapted from [9].

Contrary to valine, tryptophan is observed as monomer without added hydrogen nucleus. The complexes [trp, $\mathrm{nH}_{2} \mathrm{O}$ ] $\mathrm{n}=3-8$, are observed in the form of a double peak (figure 6). Protonated complexes could explain the second branch of those peaks. In the case of valine, protonated complexes are observed and the presence of complexes without added hydrogen nuclei remains very restricted. These results are in accord with previous studies on valine in helium droplets [8], where the weaker presence of parent ions was revealed by a close inspection of the mass region. In the study, the comparison with the gas phase result allowed to conclude that the helium droplets can partly prevent fragmentation of the parent ion.

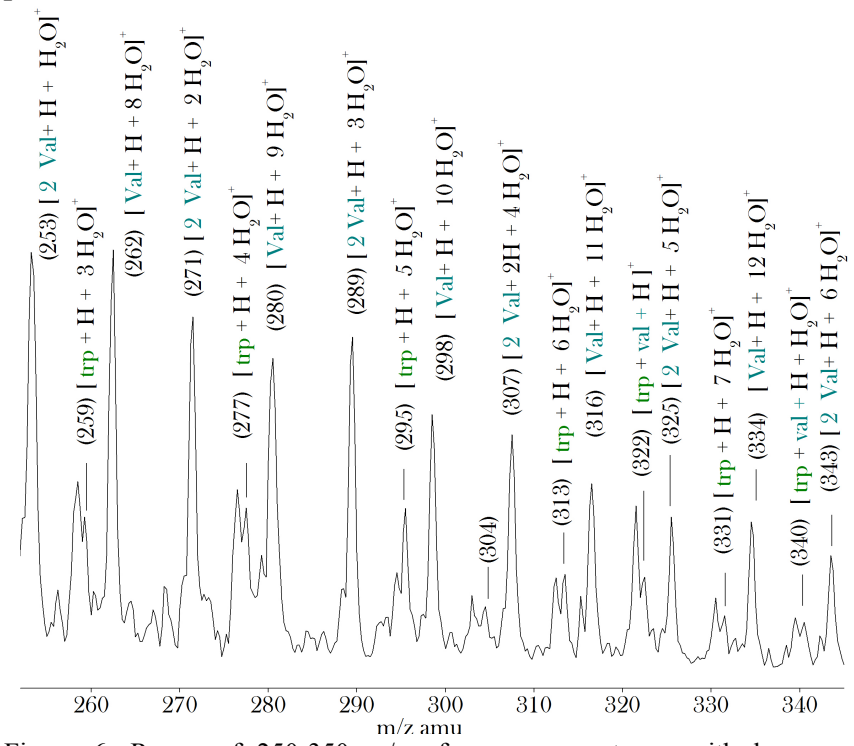

Figure 6: Range of $250-350 \mathrm{~m} / \mathrm{z}$ of a mass spectrum, with larger amplification, of embedded molecules which results from a helium droplet beam passing through the pickup cell containing both valine and tryptophan.

A heterodimer [trp,val] is detected at $321 \mathrm{~m} / \mathrm{z}$, presenting also a second peak at $322 \mathrm{~m} / \mathrm{z}$, which corresponds to the mass of [trp, val, H]. The double peak detected at 303 and $304 \mathrm{~m} / \mathrm{z}$ does not correspond to expected complexes of fragments, nor to complexes containing water molecules (figure.6). These masses represent the mass of the complexes [val, trp,] and [val, trp,H] shortened by $18 \mathrm{amu}$, which could correspond to the mass of a water molecule. The reaction of formation of a peptide bond between two amino acids also forms a water molecule as a co-product. The peptide bond formation reveals itself as an endothermic reaction by $10 \mathrm{~kJ} \mathrm{~mol}^{-1}(\Delta \mathrm{G}$ at $273 \mathrm{~K}$ in water) [14] and exhibits an activation barrier of 54-84 kJ $\mathrm{mol}^{-1}[15,16]$. In the gas phase, amino acids could also exist in the cationic form without being protonated. In this case the positive charge is located at the carbon of the carboxyl functional group. These cationic amino acids are considered to more easily form peptide bonds via the free doublet located at the C-terminal with the amine function of another amino acid [17]. A scenario in which the electron impact ionization first forms a cationic amino acid could lead to a peptide bond formation.

\section{Conclusion}

Glycine, valine, and tryptophan were embedded in helium droplets, through their vaporization in a pickup cell. The fragments of these amino acids first appeared at low temperature. These main fragments represent the amino acids shortened from the carboxyl functional group ( $-45 \mathrm{amu})$ or the amine functional group $(-73 \mathrm{amu})$. Monomers and also polymers of amino acids are detected. The cluster including a 
small number of amino acids appeared in greater quantity than the larger complexes. Ionized monomers and polymers of amino acids are able to form complexes with water molecules. The absence of water complexes with the fragments indicates an implication of the C-terminus in the formation of water complexes. Glycine forms favorably complexes with four water molecules. This suggests stable clusters of four water molecules attached to the smallest amino acids. Experiments were performed with two different amino acids in the pickup cell. The heterodimers of [tryptophan-valine] were observed and a mass corresponding to the heterodimer shortened by 18 amu was detected.

\section{Acknonowledgements}

The authors wish to thank Prof L. Wöste for his permanent encouragement. Prof P. Scheier is acknowledged for helpful discussions and for providing the helium droplet source. The authors also thank the DFG for financial support.

\section{References}

[1] Cronin J.R., Pizzarello S., Geochim. Cosmochim. Acta, 50, 2419 (1986).

[2] Elsila J. E., Glavin D. P., Dworkin J.P., Met. Plan. Sc. 44, 1323-1330, (2009).

[3] Lehmann K.K., Scoles G., Science, 279, 2065 (1998).

[4] Toennies J. P., Vilesov A. F., Ang. Chem., 43, 2622 ( 2004).

[5] Hartmann M., Lindinger A., Toennis J.P., Vilesov A.F., Chem. Phys., 239, 139 (1998).

[6] Ren Y., Kresin V.V., J. Chem. Phys. 128, 074303 (2007) doi 10.1063/1.22839602.
[7] Ferreira da Silva F., Denifl S., Märk T.D.,Ellis A.M., Scheier P., J Chem. Phys., 132, 214306 (2010).

[8] Denifl. S., Mähr I., Ferreira da Silva F., Zappa F., Märk T.D., Scheier P., Eur. Phys. J. D, 51, 73-79 ( 2009).

[9] Bellina B., Merthe D.J., Kresin V.V., J. Chem. Phys, 142, 114306 (2015).

[10] Wu C.C., Lin C-K.,Chang H-C, Jiang J-C., Kuo J-L., Klein M.L., J.Chem. Phys. 122, 074315 (2005).

[11] Heine N., PhD Thesis, FU Berlin, 2014.

[12] Lindinger A., Toennis J.P., Vilesov A.F., J.Chem.Phys., 110,1429 (1999).

[13] Ren Y., Moro R., Kresin V.V, Eur Phys. J.D, 43, 2622, (2004).

[14] Zang J., Germann M.W, Biopolymers, 95, 755-762, (2011), doi. 10.1002/bip.21642.

[15] Schroeder G.K. and Wolfenden R., Biochemistry 46, 4037 (2007), doi 10.1021/bi602600p.

[16] Sievers A., Beringer M., Rodina M.V., Wolfenden R. Proc Natl. Acad. Sci., U.S.A., 101, 7897 (2004), doi 10.73/pnasy.0401167101.

[17] Lattelais M., PhD Thesis, Université Pierre et Marie Curie, 2013. 
